\title{
Management of medically refractory trigeminal neuralgia in patients with multiple sclerosis
}

\author{
Jason S. Cheng, B.S., Rene O. Sanchez-Mejia, M.D., Mary Limbo, B.A., \\ Mariann M. Ward, R.N., M.S., AND Nicholas M. Barbaro, M.D. \\ Department of Neurological Surgery, University of California, San Francisco, California
}

\begin{abstract}
Object. Trigeminal neuralgia (TN) is a painful disorder that frequently causes lancinating, electrical shock-like pain in the trigeminal distribution. Common surgical treatments include microvascular decompression (MVD), radiosurgery, and radiofrequency ablation, and complete pain relief is generally achieved with a single treatment in 70 to $85 \%$ of cases for all modalities. In a subset of patients with multiple sclerosis (MS), however, the rates of surgical treatment failure and the need for additional procedures are significantly increased compared with those in patients without MS. In this study the authors report their experience with a cohort of 11 patients with TN who also had MS, and assess the efficacy of MVD, gamma knife surgery (GKS), and radiofrequency ablation in achieving complete or partial long-term pain relief.

Methods. Eleven patients with TN and MS who were treated by the senior author (N.B.) at the University of California, San Francisco were included in this study. All patients underwent GKS and/or radiofrequency ablation, and four received MVD. A detailed clinical history and intraoperative findings were recorded for each patient and frequent follow-up evaluations were performed, with a mean follow-up duration of 40.6 months (range 1-96 months). Pain was assessed for each patient by using the Barrow Neurological Institute scale (Scores I-V).

Conclusions. Achieving complete pain relief in patients with TN and MS required significantly more treatments compared with all other patients with TN who did not have MS ( $\mathrm{p}=0.004)$. Even when compared with a group of 32 patients who had highly refractory $\mathrm{TN}$, the cohort with MS required significantly more treatments $(\mathrm{p}=0.05)$. Radiosurgery proved to be an effective procedure and resulted in fewer retreatments and longer pain-free intervals compared with MVD or radiofrequency ablation.
\end{abstract}

\section{KEY WORDS - trigeminal neuralgia - multiple sclerosis - microvascular decompression - radiofrequency ablation - gamma knife surgery • retreatment}

Trigeminal neuralgia is a debilitating disorder affecting one or more branches of the trigeminal nerve, and is characterized by paroxysmal, shocklike pain. It occurs with a prevalence of three to five per 100,000 people in the general population and in approximately $2 \%$ of patients with MS. ${ }^{23}$ Similarly, approximately $2 \%$ of patients with TN also have MS. ${ }^{14}$ Symptoms of TN in the population with MS are more frequently bilateral, and consist of lancinating, electrical shock-like pain, which may initially be responsive to treatment with carbamazepine or gabapentin. ${ }^{12,18}$ Clinically, symptoms are indistinguishable from other cases of TN in patients without MS. Only when medical management fails are patients referred for neurosurgical treatment.

The cause of TN is unclear, although evidence supports a vascular or mass-compression hypothesis in a subset of patients. ${ }^{23}$ In patients with MS, TN was initially attributed to demyelinating lesions of the central trigeminal pathways or the trigeminal REZ, and surgical treatment frequently

Abbreviations used in this paper: $\mathrm{BNI}=$ Barrow Neurological Institute; GKS = gamma knife surgery; $\mathrm{MR}=$ magnetic resonance; MS = multiple sclerosis; MVD = microvascular decompression; $\mathrm{REZ}=$ root entry zone; $\mathrm{TN}=$ trigeminal neuralgia; $3 \mathrm{D}=$ threedimensional. consisted of percutaneous radiofrequency ablation or balloon-compression rhizolysis. ${ }^{5,6}$ In studies in which these techniques were used, complete pain relief rates reported at 1 year were as high as 86 and $90 \%$ for radiofrequency ablation and balloon-compression rhizolysis, respectively. ${ }^{10,15}$ Gamma knife surgery also yields similar success rates, and has the benefit of being noninvasive. ${ }^{24}$ Also, MVD, which was previously contraindicated in patients with MS, has returned as a surgical option after recent evidence indicating the possible coexistence of vascular compression of the REZ concomitant with the presence of a demyelinating plaque. $7,13,17,19$

Recurrence of symptoms varies widely. In patients with MS, the rate and frequency of recurrence is higher than in the general population with $\mathrm{TN}$, with a more frequent need for repeated treatments. The identification of an effective treatment modality in this unique and challenging population would likely improve symptom relief and reduce the rate and/or frequency of repeated treatments required. In this study we found GKS to be an effective treatment for refractory TN in patients with MS, resulting in lower retreatment rates and longer pain-free intervals between procedures than with radiofrequency lesioning or MVD. 


\section{CLINICAL MATERIAL AND METHODS}

\section{Patient Population}

Between 1997 and 2004, 13 patients with both MS and TN were treated with MVD, GKS, and/or radiofrequency lesioning at the Department of Neurological Surgery at the University of California, San Francisco. Before we initiated this study, permission was granted by the Committee on Human Research to conduct chart reviews and to contact patients by phone. Two patients were excluded from the study; one had received initial ablative treatment at another institution, and the diagnosis of MS was found to be mistaken in the other.

Each patient received one or more treatments consisting of MVD, GKS, or radiofrequency lesioning. Two (18\%) of the patients were men, nine $(82 \%)$ were women, and the average age was $69 \pm 8.1$ years (range 53-79 years). The mean age at symptom onset was $59 \pm 7$ years (range $40-67$ years), and the mean preoperative symptom duration was $98 \pm 35.5$ months (range 51-151 months). Compared with the other patients with TN who did not have MS, neither the age at onset $(\mathrm{p}=0.49)$ nor the preoperative symptom duration $(p=0.34)$ was significantly different. The first division of the trigeminal nerve was involved in two patients $(18 \%)$, the second division in nine $(82 \%)$, and the third division in $10(91 \%)$. Eight patients $(73 \%)$ had unilateral pain and three patients $(27 \%)$ were treated for bilateral pain (Table 1). Nine patients (82\%) also experienced numbness in one or more of the involved divisions before their last procedure. Typical symptoms included sharp, electrical shock-like pain and burning sensations that were exacerbated by eating or by touching the affected area.

Before surgery, all patients underwent medical therapy in which one or more of the following agents was used: gabapentin, carbamazepine, amitriptyline, and baclofen. Only those whose disorder was refractory to the medications (significant breakthrough pain or severe side effects) were offered surgical treatment (summarized in Table 2).

Treatment options consisted of MVD, GKS, and/or radiofrequency lesioning. All procedures were performed by the senior author (N.B.). The GKS procedure was performed in conjunction with the Department of Radiation Oncology at the University of California, San Francisco, with a dose of 70 to $85 \mathrm{~Gy}$ for the first treatment and $50 \mathrm{~Gy}$ for additional treatments. Retreatment was required in 10 patients $(91 \%)$ for refractory symptoms. For each treatment event, the patient's clinical history and any intraoperative findings or complications were recorded. Follow-up data were obtained through a combination of regular postoperative visits, email contact, and phone calls at 6 weeks and 6 months posttreatment, continuing every 6 months until complete pain resolution or retreatment. The mean followup duration was $40.6 \pm 30.5$ months (range 1-96 months), dated from the patient's first procedure.

Pain was evaluated after a patient's last procedure by using the modified BNI scale ${ }^{21}$ (Scores I-V, which are defined as follows): I, no pain; II, occasional pain not requiring medication; IIIa, no pain but continued medication; IIIb, some pain, controlled with medication; IV, some pain, not controlled with medication; and V, severe pain/no pain relief. ${ }^{22}$ A score of IIIa or better was considered to be a good outcome.

\section{RESULTS}

Patients underwent a mean of $3.8 \pm 2.6$ treatments (range one-10) with a mean period of $17 \pm 14$ months between treatments (range 1-63 months). The number of treatments received is significantly higher $(\mathrm{p}=0.004)$ compared with patients who had TN but not MS, who received a mean of $1.24 \pm 0.62$ treatments (range one to five). Even when compared with only the most refractory cases in patients with TN who required retreatment, patients with MS still underwent a significantly greater number of procedures $(\mathrm{p}=$ 0.05 ) than their counterparts without MS. Nevertheless, the time between retreatments in the two groups was not significantly different $(\mathrm{p}=0.24)$.

Six patients $(55 \%)$ received radiosurgery as a first-line treatment at a dose of 70 to $85 \mathrm{~Gy}$, whereas the remaining five $(45 \%)$ underwent radiofrequency lesioning. Ten (91\%) required retreatment because of intractable pain, as follows: none received MVD, three (30\%) received GKS, and seven $(70 \%)$ received radiofrequency lesioning. Six patients were treated a third time; none received MVD, two (33\%) received GKS, and four (67\%) received radiofrequency lesioning. For repeated GKS, patients received a reduced dose of $50 \mathrm{~Gy}$. Of the five patients who required a fourth

TABLE 1

Demographic information in 11 patients with MS who also had TN*

\begin{tabular}{|c|c|c|c|c|c|c|c|c|}
\hline \multirow[b]{2}{*}{ Case No. } & \multirow[b]{2}{*}{ Age (yrs), Sex } & \multirow[b]{2}{*}{ Age at Sx Onset } & \multirow[b]{2}{*}{ Preop Sx Duration (mos) } & \multicolumn{3}{|c|}{ Trigeminal Branch } & \multirow[b]{2}{*}{ Side } & \multirow[b]{2}{*}{$\mathrm{FU}(\mathrm{mos})$} \\
\hline & & & & $\mathrm{V}_{1}$ & $\mathrm{~V}_{2}$ & $\mathrm{~V}_{3}$ & & \\
\hline 1 & $53, \mathrm{~F}$ & 40 & 151 & yes & yes & yes & rt & 15 \\
\hline 2 & $67, \mathrm{M}$ & 50 & 130 & no & yes & yes & $\mathrm{rt}$ & 72 \\
\hline 3 & $63, \mathrm{~F}$ & 54 & 96 & no & yes (lt \& rt) & yes (lt \& rt) & bilat & 96 \\
\hline 4 & $77, \mathrm{~F}$ & 67 & 137 & no & yes & yes & lt & 56 \\
\hline 5 & $78, \mathrm{~F}$ & 67 & 120 & no & no & yes & $\mathrm{rt}$ & 1 \\
\hline 6 & $73, \mathrm{~F}$ & 64 & 52 & no & yes (lt) & yes (lt \& rt) & bilat & 55 \\
\hline 7 & $66, \mathrm{~F}$ & 61 & 65 & no & yes & no & lt & 72 \\
\hline 8 & $79, \mathrm{~F}$ & 63 & 120 & yes & yes & yes & lt & 30 \\
\hline 9 & $73, \mathrm{M}$ & UK & UK & no & no & yes (lt \& rt) & bilat & 9 \\
\hline 10 & $63, \mathrm{~F}$ & 55 & 51 & no & yes & yes & lt & 59 \\
\hline 11 & $54, \mathrm{~F}$ & 49 & 60 & no & yes & yes & lt & 13 \\
\hline
\end{tabular}

$* \mathrm{FU}=$ follow up; $\mathrm{sx}=$ symptom; $\mathrm{UK}=$ unknown; $\mathrm{V}_{1}=$ first division of the trigeminal nerve; $\mathrm{V}_{2}=$ second division of the trigeminal nerve; $\mathrm{V}_{3}=$ third division of the trigeminal nerve. 
TABLE 2

Treatment and pain relief in 11 patients with MS and $T N^{*}$

\begin{tabular}{|c|c|c|c|c|c|c|c|c|c|}
\hline \multirow[b]{2}{*}{ Case No. } & \multicolumn{6}{|c|}{ Treatments } & \multirow[b]{2}{*}{ BNI Score } & \multirow[b]{2}{*}{ Pain-Free w/ Meds } & \multirow[b]{2}{*}{$\%$ Pain Relief } \\
\hline & $1 \mathrm{st}$ & $2 \mathrm{nd}$ & $3 \mathrm{rd}$ & 4 th & 5 th & 6 th & & & \\
\hline 1 & GKS & $\mathrm{RF}$ & - & - & - & - & I & yest & 100 \\
\hline 2 & $\mathrm{RF}$ & $\mathrm{RF}$ & GKS & MVD/R & GKS & $\mathrm{RF}$ & IIIb & no & 50 \\
\hline 3 & RF (lt) & GKS (lt) & RF (lt) & MVD/R (lt) & $\mathrm{RF}(\mathrm{rt})$ & RF (lt) & IIIb & no & 25 \\
\hline 4 & GKS & $\mathrm{RF}$ & RF (lt) & GKS & - & - & IIIa & yes & 100 \\
\hline 5 & $\mathrm{RF}$ & $\mathrm{RF}$ & - & - & - & - & IIIb & no & 25 \\
\hline 6 & GKS (lt) & $\mathrm{RF}(\mathrm{rt})$ & $\mathrm{RF}(\mathrm{rt})$ & MVD (lt) & RF (lt) & RF (lt) & IIIa & yes & 100 \\
\hline 7 & GKS & - & - & - & - & - & IIIa & yes & 100 \\
\hline 8 & GKS & GKS (lt) & $\mathrm{RF}$ & MVD/R & - & - & IIIb & no & 25 \\
\hline 9 & RF (lt) & $\mathrm{RF}(\mathrm{rt})$ & - & - & - & - & IIIb & no & 50 \\
\hline 10 & GKS & GKS & - & - & - & - & IIIa & yes & 100 \\
\hline 11 & $\mathrm{RF}$ & $\mathrm{RF}$ & GKS & - & - & - & IIIa & yes & 100 \\
\hline
\end{tabular}

$*$ Meds = medications; MVD/R = MVD with partial rhizotomy, RF = radiofrequency lesioning, $-=$ no further treatments.

$\dagger$ The patient was able to taper off all medications while remaining pain free.

treatment, three $(60 \%)$ received MVD with partial rhizotomy, one (20\%) received MVD only, and one (20\%) received repeated GKS. Of the four patients who underwent MVD as their fourth treatment, three (75\%) required two additional treatments before obtaining pain control, and only one $(25 \%)$ experienced complete pain relief after MVD while continuing to receive a reduced dosage of medication. Vascular compression of the REZ was found in three patients who underwent MVD; the superior cerebellar artery was involved in two patients and a small vein in one.

Overall, nine patients $(82 \%)$ received GKS as one of their treatments and another nine (82\%) received radiofrequency lesioning. Only four (36\%) underwent MVD after repeated failures of GKS, radiofrequency lesioning, or both. As shown in Table 3, the rate of retreatment was $63 \%$ for GKS, $71 \%$ for radiofrequency lesioning, and $75 \%$ for MVD. Gamma knife surgery resulted in the longest mean time between treatments (35 \pm 27 months, range 12-75 months). Radiofrequency lesioning provided a mean of $29 \pm 27$ months of relief (range 1-75 months), and MVD yielded $18 \pm 7$ months of relief (range 11-24 months). Nevertheless, given the small sample size, the differences in the aforementioned values were not statistically significant.

Outcomes were assessed at 12 months after the last procedure according to the BNI criteria. One patient (Case 1) experienced complete pain relief not requiring medication (BNI Score I) after radiofrequency lesioning, whereas five additional patients (45\%) were pain free but continued their medications at reduced dosages (BNI Score IIIa). Of those, four (Cases 4, 7, 10, and 11) received GKS and one (Case 6) underwent radiofrequency lesioning as their last treat-

\section{TABLE 3}

Percent receiving retreatment according to initial modality and time to next procedure*

\begin{tabular}{cccc}
\hline \hline Tx Received & $\begin{array}{c}\text { No. of } \\
\text { Patients }\end{array}$ & $\begin{array}{c}\% \\
\text { Retreated }\end{array}$ & Mean Time Before Next Tx (mos) \\
\hline GKS & 9 & 63 & 35 \\
RF & 9 & 71 & 29 \\
MVD & 4 & 75 & 18 \\
\hline
\end{tabular}

* Each time a treatment was performed counts as a separate event. The mean time before next treatment is an estimate of the pain-free interval resulting from a particular treatment event. Abbreviation: $t x=$ treatment. ment. The remaining five patients $(45 \%)$ experienced moderate pain relief with a mean pain reduction of $33 \pm 14 \%$ (range 25-50\%) while still taking medication 12 months after the last procedure (Table 4). In these patients the medication dosage was reduced compared with preoperative regimens and the pain was controlled (BNI Score IIIlb).

Based on the last treatment received, GKS accounted for four of the six patients with BNI scores of IIIa or better. The remaining two patients received radiofrequency lesioning as their final treatment and MVD did not yield a Score IIIa outcome in any patient. Preoperative symptom duration was also related to pain outcome; of the six individuals with BNI Score IIIa or better, four had the shortest preoperative symptom durations (51, 52, 60, and 65 months), whereas the remaining two patients had longer symptom durations (137 and 151 months). Unlike in previous studies, preoperative sensory deficits did not affect outcome; five $(83 \%)$ of six patients who became pain-free experienced sensory deficits in one or more of the affected divisions prior to their final procedure. ${ }^{15}$

A Kaplan-Meier survival curve was plotted to compare retreatment rates after GKS and radiofrequency lesioning with time from first treatment. Microvascular decompression was not included because no patient received it as the initial treatment. Similar rates of retreatment were observed between both modalities, with all patients who underwent radiofrequency lesioning and six $(86 \%)$ of seven who underwent GKS requiring additional treatment (Fig. 1). There were no complications in any of the patients.

\section{DISCUSSION}

The fact that a significant percentage of patients with MS have TN that is clinically indistinguishable from the type of TN that is unrelated to MS has led investigators to postulate

TABLE 4

Medication usage after last procedure

\begin{tabular}{lccccc}
\hline \hline \multicolumn{1}{c}{ Subgroup } & $\begin{array}{c}\text { No } \\
\text { Meds }\end{array}$ & $\begin{array}{c}\text { Reduced } \\
\text { Meds }\end{array}$ & $\begin{array}{c}\text { Same } \\
\text { Meds }\end{array}$ & $\begin{array}{c}\text { Increased } \\
\text { Meds }\end{array}$ & Final Tx \\
\hline pain-free & 1 & 5 & 0 & 0 & 4 GKS, 2 RF \\
reduced pain & 0 & 5 & 0 & 0 & 1 MVD, 4 RF \\
\hline
\end{tabular}


a common source of pain in the portion of the trigeminal nerve that contains central myelin. ${ }^{20}$ Nevertheless, the fact that patients with both TN and MS are much less likely to attain long-term pain relief with a single surgical treatment indicates that these two groups of patients have significant differences in the pathophysiological origins of their condition. ${ }^{1,11}$ One possibility is that patients with MS may have extension of their pathological features into the brainstem and away from areas treated by surgical approaches. ${ }^{16}$ The recurrence rate in these patients is significantly higher, however, even when treatment is directed away from the trigeminal REZ, as is done in percutaneous techniques. ${ }^{1}$ Treatment of TN in patients with MS has met with difficulty in the past because of uncertainty about the cause of TN in this group. Recent literature favors multiple causes, including the possibility of both central MS lesions of the trigeminal pathway and peripheral vascular compression of the trigeminal REZ as causes that are not mutually exclusive., ${ }^{4,-9}$

Initial studies examining the role of MVD for treatment of patients with both MS and TN have yielded mixed results. Resnick, et al., ${ }^{20}$ examined five patients who had previously undergone unsuccessful percutaneous procedures, and reported better outcomes in two who had received MVD with partial rhizotomy compared with MVD alone. In each instance, vascular compression of the superior cerebellar artery was noted intraoperatively. These results indicate that destructive therapies may yield better results in patients with MS, although these therapies have an increased potential for producing more severe paresthesias. In a study by Broggi, et al., ${ }^{7}$ conducted in a cohort of 10 patients with both TN and MS who underwent MVD, the authors found vascular compression of the trigeminal REZ in all patients, and nine were free of pain immediately postoperatively. In a follow-up period averaging 24 months per patient (range 12-39 months), five continued to experience complete pain relief and required no medications. Overall, the proportion of patients experiencing complete pain relief was lower in those with both MS and TN (50\% compared with 74\%) than in a cohort of patients with idiopathic TN in the same series.

A subsequent study by the same authors ${ }^{8}$ described the results in 35 patients with MS who underwent MVD. Vascular compression of the trigeminal nerve was observed in 16 patients $(46 \%)$, and in $26(74 \%)$ demyelinating lesions of the brainstem trigeminal pathways were seen on MR imaging. Severe vascular compression was also correctly predicted based on MR imaging in 11 of 12 patients. Longterm outcomes after a mean follow-up duration of 44 months (range 6-108 months) revealed complete pain relief without medication in $39 \%$ and pain relief with intermittent use of medication in an additional $14 \%$ of patients. Although these studies highlight the potential therapeutic value of MVD in patients with MS, the results are far worse than those typically reported for patients without MS. In our cohort, MVD with or without partial rhizotomy did not prove to be an effective treatment modality, resulting in the shortest time to the next procedure and never achieving a BNI score of IIIa or better.

In an effort to improve MVD outcomes in patients with MS, Akimoto, et al., ${ }^{2}$ reconstructed high-resolution 3D models from 3D constructive interference in steady state and 3D fast imaging with steady-state precession MR images to predict vascular involvement preoperatively in a co- hort of 24 patients. The predictions were verified intraoperatively, and in 23 cases (96\%) the imaging enabled correct identification of the vessel affecting the trigeminal nerve. In the other case, the vessel was a small branch of the superior cerebellar artery that was not visible on imaging. In the future, the ability to visualize vascular compression on high-resolution MR imaging will likely be the deciding factor when selecting patients (both with and without MS) for MVD.

Although none of the procedures used in our small study proved to be statistically more effective than the others in patients with MS, GKS and to a lesser extent radiofrequency lesioning yielded longer intervals between procedures than MVD with or without partial rhizotomy. This indicates that destructive lesioning in the population of patients with both MS and TN may produce better outcomes. Although the goal of any treatment is to minimize paresthesias while maximizing pain relief, evidence indicates that there is a close correlation between the two, with higher levels of paresthesias correlating with greater pain relief. ${ }^{15}$ In addition, all four patients in our study who received GKS as the final treatment attained a BNI score of IIIa or better at 12 months. In one instance (Case 7), GKS was also effective in controlling pain (Score IIIa) after the first treatment. Although Kaplan-Meier analysis did not reveal significantly better outcomes for GKS when compared with radiofrequency lesioning, this is likely due to the small sample size in the study. Radiosurgery has always been an attractive option in high-risk surgical candidates, and its low rate of complications along with the option of repeating the procedure enhances its appeal. Response rates as high as $80 \%$ have been achieved in patients with MS and TN. In that analysis of 15 patients with MS, $12(80 \%)$ reported pain relief within a mean follow-up period of 17 months (range 6-38 months). ${ }^{22}$ It is interesting to speculate that GKS may have a unique role in the treatment of patients with MS and $\mathrm{TN}$ because the radiation can be delivered to the brainstem portion of the REZ, something that is not possible with other surgical treatments.

In a separate study examining single- or dual-isocenter therapy and increasing dose in a group of 63 patients with

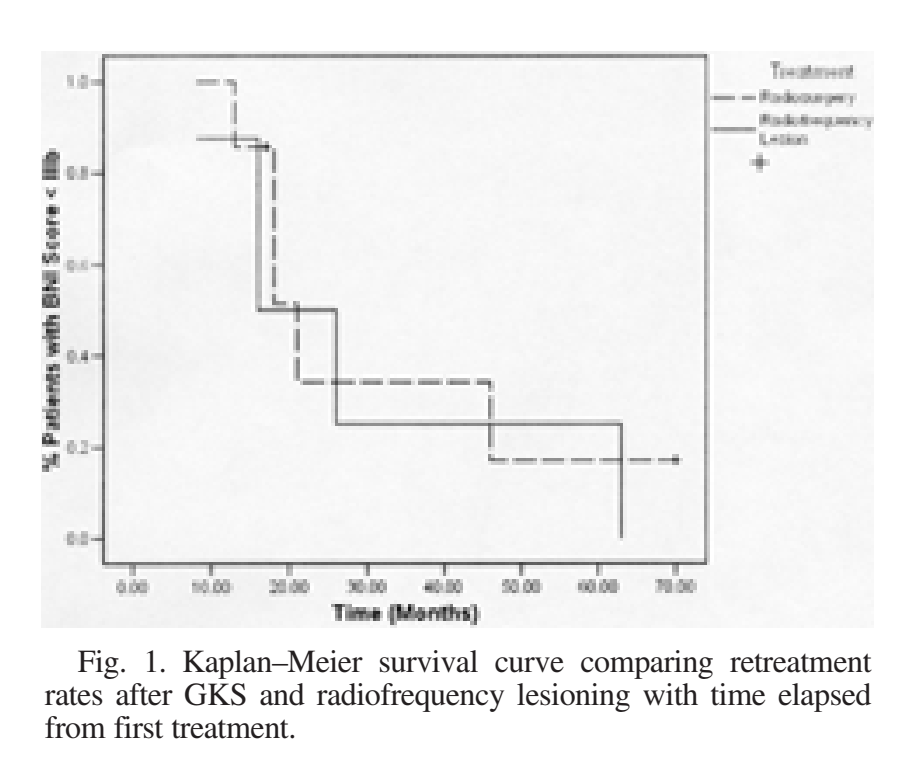

Neurosurg. Focus / Volume 18 / April, 2005 
TN (10 with MS), the authors reported greater improvement in symptoms after using dual- compared with single-isocenter therapy, and they also found higher doses (maximum 90 Gy) to be more effective. ${ }^{3}$ Only five patients (8\%) experienced mild facial numbness from the treatment. Thus, GKS remains an important treatment modality in patients with both MS and TN, and studies are needed to determine whether this group of patients should receive modified treatment regimens.

In addition to GKS, percutaneous radiofrequency lesioning has also been used with success in patients with MS, and in one study a $94.1 \%$ success rate was reported in a cohort of 17 patients with MS and TN..$^{15}$ Fourteen of the patients were free of pain and had discontinued medication during the follow-up period, and the remaining three required medication at lower doses. Seventy-six percent of patients reported hypalgesia and/or hypesthesia. In a separate study of 179 patients, a $75 \%$ hypesthesia rate was also reported after percutaneous radiofrequency rhizotomy, an indication of the increased morbidity associated with radiofrequency lesioning. In our study, radiofrequency lesioning did not prove to be as effective a treatment as GKS; only one $(11 \%)$ of nine patients experienced complete pain relief after therapy and did not require additional treatment. Nevertheless, in this group of patients a minimally invasive treatment that offers immediate pain relief will likely remain on the list of surgical alternatives.

\section{CONCLUSIONS}

Surgical treatment of TN in patients with MS frequently results in lower success rates than in patients without MS. Treatments that achieve long-term pain-free intervals with minimal morbidity are needed for these patients. Data from our study indicate that GKS is an efficacious treatment for patients with MS and refractory TN. It has a low risk of complications and a higher proportion of longer pain-free intervals compared with radiofrequency lesioning and MVD. Future studies should concentrate on whether the dose to the brainstem plays a significant role in the longterm efficacy of GKS and whether neuroimaging studies can be used to predict which patients with MS may have vascular compression, thereby making them better candidates for MVD.

\section{Acknowledgments}

We thank Kathleen Lamborn and Sharon Reynolds for their contribution to this work.

\section{References}

1. Adams CB: Trigeminal neuralgia: pathogenesis and treatment. Br J Neurosurg 11:493-495, 1997

2. Akimoto H, Nagaoka T, Nariai T, et al: Preoperative evaluation of neurovascular compression in patients with trigeminal neuralgia by use of three-dimensional reconstruction from two types of high-resolution magnetic resonance imaging. Neurosurgery 51:956-962, 2002

3. Alpert TE, Chung CT, Mitchell LT, et al: Gamma knife surgery for trigeminal neuralgia: improved initial response with two isocenters and increasing dose. J Neurosurg 102 (Suppl): $185-188,2005$

4. Barker FG, Jannetta PJ, Bissonette DJ, et al: The long-term out- come of microvascular decompression for trigeminal neuralgia. New Engl J Med 334:1077-1083, 1996

5. Belber CJ, Rak RA: Balloon compression rhizolysis in the surgical management of trigeminal neuralgia. Neurosurgery 20: 908-913, 1987

6. Brisman R: Trigeminal neuralgia and multiple sclerosis. Arch Neurol 44:379-381, 1987

7. Broggi G, Ferroli P, Franzini A, et al: Microvascular decompression for trigeminal neuralgia: comments on a series of 250 cases, including 10 patients with multiple sclerosis. J Neurol Neurosurg Psychiatry 68:59-64, 2000

8. Broggi G, Ferroli P, Franzini A, et al: Operative findings and outcomes of microvascular decompression for trigeminal neuralgia in 35 patients affected by multiple sclerosis. Neurosurgery 55: 830-839, 2004

9. Broggi G, Ferroli P, Franzini A, et al: Role of microvascular decompression in trigeminal neuralgia and multiple sclerosis. Lancet 354:1878-1879, 1999

10. Brown JA, McDaniel MD, Weaver MT: Percutaneous trigeminal nerve compression for treatment of trigeminal neuralgia: results in 50 patients. Neurosurgery 32:570-573, 1993

11. Fromm GH, Terrence CF, Maroon JC: Trigeminal neuralgia Current concepts regarding etiology and pathogenesis. Arch Neurol 41:1204-1207, 1984

12. Hooge JP, Redekop WK: Trigeminal neuralgia in multiple sclerosis. Neurology 45:1294-1296, 1995

13. Jannetta PJ: Arterial compression of the trigeminal nerve at the pons in patients with trigeminal neuralgia. J Neurosurg 26 (Suppl):159-162, 1967

14. Jensen TS, Rasmussen P, Reske-Nielsen E: Association of trigeminal neuralgia with multiple sclerosis: clinical and pathological features. Acta Neurol Scand 65:182-189, 1982

15. Kanpolat Y, Berk C, Savas A, et al: Percutaneous controlled radiofrequency rhizotomy in the management of patients with trigeminal neuralgia due to multiple sclerosis. Acta Neurochir 142:685-690, 2000

16. Kerr FW: Pathology of trigeminal neuralgia: light and electron microscopic observations. J Neurosurg 26 (Suppl):151-156, 1967

17. Love S, Gradidge T, Coakham HB: Trigeminal neuralgia due to multiple sclerosis: ultrastructural findings in trigeminal rhizotomy specimens. Neuropathol Appl Neurobiol 27:238-244, 2001

18. Moulin DE, Foley KM, Ebers GC: Pain syndromes in multiple sclerosis. Neurology 38:1830-1834, 1988

19. Olafson RA, Rushton JG, Sayre GP: Trigeminal neuralgia in a patient with multiple sclerosis. An autopsy report. J Neurosurg 24: 755-759, 1966

20. Resnick DK, Jannetta PJ, Lunsford LD, et al: Microvascular decompression for trigeminal neuralgia in patients with multiple sclerosis. Surg Neurol 46:358-362, 1996

21. Rogers CL, Shetter AG, Fiedler JA, et al: Gamma knife radiosurgery for trigeminal neuralgia: the initial experience of The Barrow Neurological Institute. Int J Radiat Oncol Biol Phys 47: 1013-1019, 2000

22. Rogers CL, Shetter AG, Ponce FA, et al: Gamma knife radiosurgery for trigeminal neuralgia associated with multiple sclerosis. J Neurosurg 97 (Suppl 5):529-532, 2002

23. Sweet WH: The treatment of trigeminal neuralgia (tic douloureux). N Engl J Med 315:174-177, 1986

24. Urgošik D, Vymazal J, Vladyka V, et al: Gamma knife treatment of trigeminal neuralgia: clinical and electrophysiological study. Stereotact Funct Neurosurg 70 (Suppl 1):200-209, 1998

Manuscript received March 23, 2005.

Accepted in final form March 25, 2005.

Address reprint requests to: Nicholas M. Barbaro, M.D., Department of Neurological Surgery, University of California, San 